\title{
Quantification of human body fat tissue percentage by MRI
}

\author{
Hans-Peter Müller ${ }^{a *}$, Florian Raudies ${ }^{b}$, Alexander Unrath ${ }^{a}$, \\ Heiko Neumann ${ }^{b}$, Albert C. Ludolph ${ }^{a}$ and Jan Kassubek ${ }^{a}$
}

\begin{abstract}
The MRI-based evaluation of the quantity and regional distribution of adipose tissue is one objective measure in the investigation of obesity. The aim of this article was to report a comprehensive and automatic analytical method for the determination of the volumes of subcutaneous fat tissue (SFT) and visceral fat tissue (VFT) in either the whole human body or selected slices or regions of interest. Using an MRI protocol in an examination position that was convenient for volunteers and patients with severe diseases, 22 healthy subjects were examined. The software platform was able to merge MRI scans of several body regions acquired in separate acquisitions. Through a cascade of image processing steps, SFT and VFT volumes were calculated. Whole-body SFT and VFT distributions, as well as fat distributions of defined body slices, were analysed in detail. Complete three-dimensional datasets were analysed in a reproducible manner with as few operator-dependent interventions as possible. In order to determine the SFT volume, the ARTIS (Adapted Rendering for Tissue Intensity Segmentation) algorithm was introduced. The advantage of the ARTIS algorithm was the delineation of SFT volumes in regions in which standard region grow techniques fail. Using the ARTIS algorithm, an automatic SFT volume detection was feasible. MRI data analysis was able to determine SFT and VFT volume percentages using new analytical strategies. With the techniques described, it was possible to detect changes in SFT and VFT percentages of the whole body and selected regions. The techniques presented in this study are likely to be of use in obesity-related investigations, as well as in the examination of longitudinal changes in weight during various medical conditions. Copyright (C) 2010 John Wiley \& Sons, Ltd.
\end{abstract}

Keywords: MRl; adipose tissue; subcutaneous fat; visceral fat; whole body

\section{INTRODUCTION}

Interest has been increasing in the investigation of body fat distribution following the complications of obesity from the 1980 s onwards. The accumulation of body fat in the abdominal area has been reported in various diseases commonly associated with obesity (1-4). However, the quantification of body fat and its distribution is particularly difficult (5). Sufficient accuracy has been achieved using techniques such as ultrasound or the measurement of skin-fold thickness by callipers (6-10). These measurements provide a simple and useful estimation of the proportion of abdominal fat (11), but are unable to distinguish between visceral fat tissue (VFT) and abdominal subcutaneous fat tissue (SFT). However, internal adipose tissue compartments are difficult to measure accurately. There is evidence that mainly VFT, which is morphologically and functionally different from SFT, is associated with insulin resistance, hyperinsulinaemia, dyslipidaemia and hypertension (12).

Beginning in the 1990s, advances in computed tomography $(8,13,14)$ and MRI $(15-17)$ allowed for new possibilities in the investigation of subcutaneous, intraperitoneal and visceral adipose tissue components (5). These imaging techniques enabled VFT and SFT to be distinguished in each recorded slice (12). The accuracy of both modalities has been validated against autopsy in humans (5). Nevertheless, because of the inherent drawback of exposure to ionising radiation for volunteers in computed tomography, MRI is the preferred technique $(18,19)$. In addition to these techniques, recent fat-related studies concern- ing the presence and functionality of brown adipose tissue have been the topic of research with positron emission tomography (20-22).

Among the spectrum of MRI-based techniques, $\mathrm{T}_{1}$-weighted imaging (spin-echo as well as gradient-echo) $(18,19)$ has been proven to show 'bright' fat because of its reduced relaxation time: $T_{1}$ of fat is about 300 ms versus approximately $1000 \mathrm{~ms}$ of most water-containing tissues (23).

Several studies have correlated the results from MRI fat distribution analysis with medical indices, e.g. cardiovascular risk factors (24) and diabetes (25), and have made comparisons with other methods of estimation of total and intra-abdominal fat distribution, e.g. Siegel et al. (26).

None of these studies has made use of whole-body MRI scans to achieve these results. The first results not arising merely from

\footnotetext{
* Correspondence to: H.-P. Müller, c/o Prof. Dr. Jan Kassubek, Department of Neurology, University of UIm, D-89081 Ulm, Germany.

E-mail: hans-peter.mueller@uni-ulm.de; E-mail: jan.kassubek@uni-ulm.de

a H.-P. Müller, A. Unrath, A. C. Ludolph, J. Kassubek

Department of Neurology, University of UIm, Ulm, Germany

b F. Raudies, H. Neumann

Institute of Neural Information Processing, University of UIm, Ulm, Germany
}

Abbreviations used: ARTIS, Adapted Rendering for Tissue Intensity Segmentation; ATLAS, Automatic Tissue Labelling Analysis Software; SFT, subcutaneous fat tissue; VFT, visceral fat tissue. 
an analysis of the amount of adipose tissue, but from a detailed analysis of whole-body topography, were published in 2005 by Machann et al. (12). Recent MRI studies have dealt with the assessment of abdominal adipose tissue (27), automated segmentation of VFT and SFT $(28,29)$, and segmentation of intermuscular adipose tissue (30), as well as with pre-processing techniques, e.g. the automatic correction of inhomogeneities in intensity (31).

Inspired by these investigations, an MRI protocol was set up which uses an examination position convenient for volunteers and patients with severe medical conditions. A new software platform was developed that was able to merge MRI scans of several body regions from separate acquisitions. The software was set up to analyse whole-body fat distributions, as well as fat distributions of single body regions or slices of interest. The novelty of this work is that complete three-dimensional datasets, as well as defined body parts, can be analysed in a reproducible manner with as few operator interventions as possible.

\section{METHODS}

\section{Scanning protocol}

MRI data were acquired on a 1.5 T scanner (Symphony, Siemens Medical, Erlangen, Germany). The bore size was $60 \mathrm{~cm}$, horizontal. A quantum gradient system with a gradient field strength up to $30 \mathrm{mT} / \mathrm{m}$ (52 mT/m effective) was available with a slew rate of up to $125 \mathrm{~T} / \mathrm{m} / \mathrm{s}$ ( $216 \mathrm{~T} / \mathrm{m} / \mathrm{s}$ effective).

The whole-body MRI scan was recorded by the acquisition of six to eight $T_{1}$-weighted volumes (standard $T_{1}$-weighted turbo spin-echo sequence), each consisting of 36 two-dimensional slices (slice thickness, $5 \mathrm{~mm} ; 1 \mathrm{~mm}$ gap). The in-plane resolution was $1.8 \mathrm{~mm} \times 1.3 \mathrm{~mm}(206 \times 384$ voxels); 206 phase-encoding steps were used and the phase-encoding direction was anterior to posterior. The flip angle was $160^{\circ}$, the repetition time was $476 \mathrm{~ms}$ and the time to echo was $12 \mathrm{~ms}$. The acquisition time for one volume was $4.5 \mathrm{~min}$, resulting in a total acquisition time between 27 and $36 \mathrm{~min}$. To confirm that no gap was left between the consecutive volumes, an overlap of about $6-18 \mathrm{~mm}$ (see 'Registration - merging of the single volumes') was chosen between the volumes, so that a total area of at least $120 \mathrm{~cm}$ was scanned.

\section{Subject population}

Twenty healthy volunteers (male to female ratio, 10: 10; average age, $66.2 \pm 8.2$ years for men and $66.5 \pm 7.4$ years for women) underwent the MRI scanning protocol. In addition, in order to test the reproducibility of the scanning and analysis results, one male ( 25 years) and one female ( 22 years) volunteer were scanned at three consecutive days within 1 week.

\section{RESULTS}

Data pre-processing and analysis were performed by the inhouse-developed software package ATLAS (Automatic Tissue Labelling Analysis Software). For fat percentage determination, the abdominal region between the abdomen above the diaphragm and the thigh, covering about $65 \mathrm{~cm}$ depending on the subject's height (range, $56-72 \mathrm{~cm}$ ), was selected.

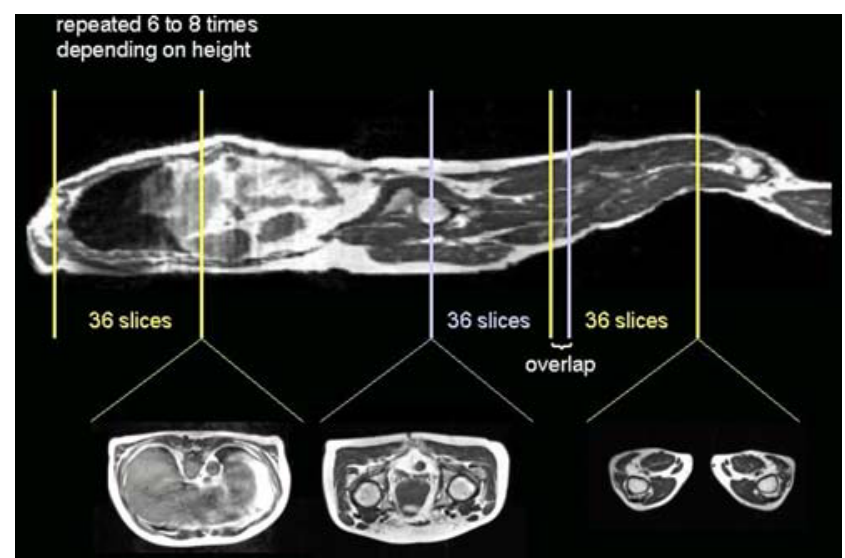

Figure 1. Merging of separately recorded volumes (each consisting of 36 slices) to one contiguous whole-body volume. In order to avoid gaps, an overlap between two subsequently recorded volumes was defined (data from a 64-year-old male volunteer, subject 1 ).

\section{Registration - merging of the single volumes}

Each of the single recorded three-dimensional volumes covered a body section of about $22 \mathrm{~cm}$. Depending on the subject's height, six to eight volumes of this size were recorded. In between two consecutively recorded three-dimensional volumes, an overlap of between 6 and $18 \mathrm{~mm}$ (one to three slices) allowed the application of a conjugated simplex fitting method with three degrees of freedom $(x, y, z$ translational shift) (32). In this way, one contiguous body volume was merged (Fig. 1). The intensity of a voxel at position $\vec{x}$ in the merged volume was defined by:

$$
I(\vec{x})=l_{j}\left(\vec{v}+\vec{t}_{j}\right)
$$

where $l_{j}\left(\vec{v}+\vec{t}_{j}\right)$ is the corresponding intensity at position $\vec{v}$ in volume $j$ shifted by the translation vector $\vec{t}_{j}$. The translation vector $\vec{t}_{j}$ was determined by the fitting procedure for two subsequent volumes. As $\vec{t}_{j}$ did not usually fit exactly the slice dimension of $6 \mathrm{~mm}$, linear nearest-neighbour interpolation was used for $I(\vec{x})$ of the merged volume. In the overlapping regions, the intensity of the merged volume was calculated by linear nearest-neighbour interpolation of the intensities of the two overlapping volumes. If a scanner is used which contains the geometrical position information, no overlap recording is needed. Simple transformation solves the merging problem. Prior to subsequent analysis, the data were supersampled to isotropic voxels with a size of $1.2 \times 1.2 \times 1.2 \mathrm{~mm}^{3}$.

\section{Intensity homogenisation}

In cases in which magnetic field or gradient inhomogeneities or distortions might cause image inhomogeneities over the large scanning area (Fig. 2a, left), an interactive repair functionality was used in order to correct the distortions and prepare the dataset for further data operations. The algorithm increases the image intensity by a factor of 1.2 in operator-selected three-dimensional regions, where the shape of the processed region could be chosen depending on the size and shape of the distortion. An example is shown in Fig. 2a (right). 

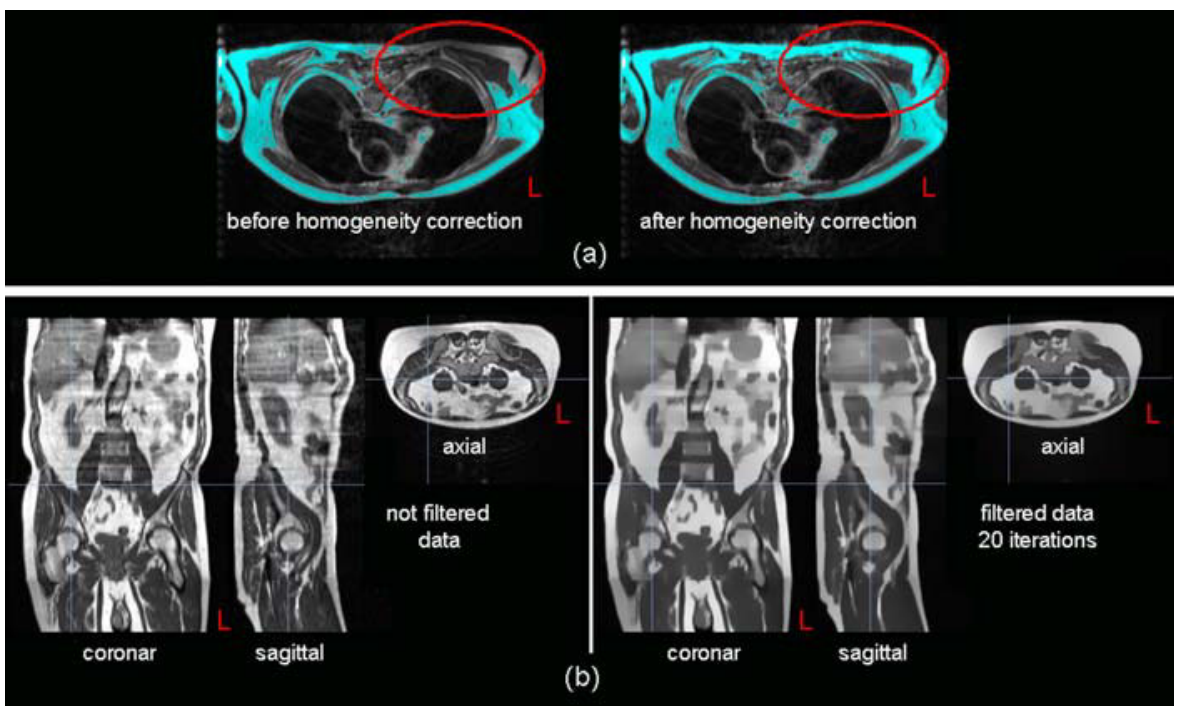

Figure 2. (a) Image pre-processing - axial views. Images before (left) and after (right) correction for inhomogeneities - the data are thresholded in light blue for better visualisation. (b) A comparison between the input volume and the volume homogenised by diffusion filtering with 20 iterations (coronal, sagittal, axial views, see text for further details) (data from a 64 -year-old male volunteer, subject 1 ).

\section{Diffusion filtering of volumetric data}

Diffusion filtering is motivated by the physical process of heat transfer through different tissues and the boundaries between them. There is a twofold analogy in image processing to this physical process: the intensity value of the voxels is the initial activation level, or heat, with which the physical process starts (initial value condition), and the media and their boundaries are defined by certain structures given within the volume of voxels. Formally, heat or activity transfer is described by $(33,34)$ :

$$
\begin{gathered}
\partial_{t} u=\nabla(\rho \cdot \nabla u), \text { with } u=u(\vec{x}, t) \in \Re \\
\text { and } \rho=\rho(\vec{x}, t) \in \Re
\end{gathered}
$$

neglecting convection and external forces [Fick's second law (35)]. Here, $u$ denotes the current activity and $\rho$ the properties, i.e. the diffusibility, of the medium. The gradient operator $\nabla$ is defined with respect to the three spatial dimensions $x, y$ and $z$. The activity corresponding to the smoothed intensity level is propagated using $\rho$ as a local steering variable over time. Intuitively, $\rho$ defines barriers, or boundaries, to locally gate the process of intensity diffusion. Different variants of eqn (2) can be instantiated depending on the particular format and structure of the diffusibility denoted in $\rho$. Here:

$$
\rho=\frac{1}{1+(\|\nabla u\| / \lambda)^{2}}
$$

was chosen with $\lambda=3.5 / 255$, given an input intensity range of $[0,1]$. This defines a nonlinear, isotropic and thus inhomogeneous diffusion process, as $\rho$ is a scalar function which itself is a function of $u$. Together, this leads to the spatial inhomogeneity of the diffusibility in the grid of voxels. This diffusion mechanism enforces the condition that regions of similar intensity (low gradient, $\|\nabla u\| \approx 0$ ) are homogenised, whereas sharp boundaries of intensities still persist (high gradient, $\|\nabla u\|>>0$ ). An example of this diffusion processing, with representative slices, is shown in Fig. 2b. In general, diffusion processing depends on the time over which the physical process lasts. A numerical approximation of the diffusion process discretises time into small intervals counted by iterations. With respect to postprocessing, the duration of diffusion is critical in the sense that heat should be not fully but 'sufficiently' homogenised, so that the subsequent segmentation is constant with respect to the applied number of iterations.

The reasons for diffusion homogenisation and differences between data before and after homogenisation are presented in Results.

\section{Segmentation of SFT}

SFT was identified by a two-level, intensity-based threshold algorithm indicating the voxels that could contribute to SFT. The algorithm detected voxels within an operator-defined intensity range, followed by an automatic identification of SFT voxels. In order to be defined as subcutaneous, the single voxel had to be adjacent to the body surface in a first step. In a further iterative process, voxels that had an intensity within the intensity range and were directly adjacent to a voxel which had already been defined as subcutaneous were also defined as SFT. Similar algorithms have been used by Machann et al. (12). An improvement was achieved by the application of the ARTIS (Adapted Rendering for Tissue Intensity Segmentation) algorithm. A plane was set up perpendicular to the coronal direction; then, for each coronal voxel row, the subject border was searched independently. If at least three subsequent voxels had an intensity in between the thresholds, this voxel position was defined as a border voxel, and all voxels along the row that had an intensity in between the thresholds were defined as SFT, until one voxel was found outside the threshold range. This distance (number of voxels) was defined as the SFT thickness for this voxel row (Fig. 3a). In order to ensure that single voxel rows did not reveal volatile or nonrealistic results, the algorithm did not work independently for each row, but used the median values in the two-dimensional vicinity of this voxel row for the border voxel position, as well as for the SFT thickness for this voxel row. The 


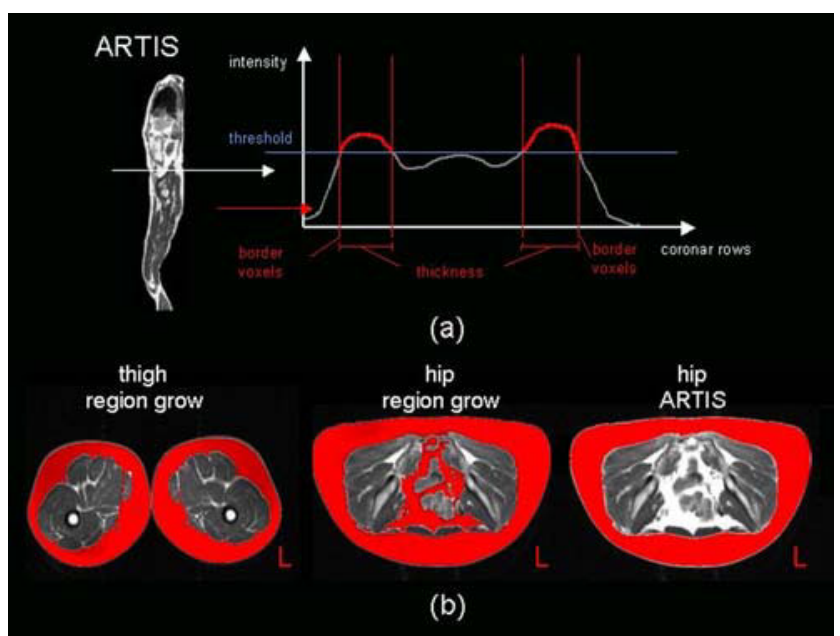

Figure 3. (a) Schematic illustration of the determination of the border voxels and the thickness for one voxel row as performed using the ARTIS (Adapted Rendering for Tissue Intensity Segmentation) algorithm. If at least three subsequent voxels have an intensity in between the thresholds, this voxel position is defined as a border voxel. All voxels along the row that have an intensity in between the thresholds are defined as subcutaneous fat tissue (SFT) as long as one voxel is not within the threshold range. This length is defined as the SFT thickness for this voxel row. (b) Region grow versus ARTIS algorithm. Although a region grow algorithm works quite well, e.g. in the thigh (left), intensity bridges lead to errors in other regions, e.g. the hip (centre). The ARTIS algorithm overcomes these problems (right) (data from a 64-year-old male volunteer, subject 1).

dimension of the vicinity was defined as 20 voxels in each dimension.

Although a region grow algorithm works quite well, e.g. in the thigh (Fig. 3b, left), intensity bridges lead to errors in other regions, e.g. the hip (Fig. 3b, centre). The ARTIS algorithm overcomes these problems (Fig. 3b, right). To test the stability of the algorithms and in order to avoid errors caused by the interactively adjusted threshold, an automatic procedure for the determination of the intensity thresholds for the whole-body volume, as well as for the fat volumes, was developed. The SFT determination was performed depending on the intensity threshold (for the data in this study, the intensity range was 0-4095). The threshold was varied in steps of 50 units (i.e. approximately $1 \%$ of the intensity maximum). The resulting relationship between the threshold and the number of voxels is displayed in Fig. 4a. The characteristics of the ARTIS algorithm (which was optimised for subcutaneous fat determination) revealed stability in the threshold range (at an intensity threshold of about 1400, depending on the subject data) in which the SFT volume was determined. Here, the second derivative equals zero (Fig. 4a). Over an intensity threshold range of \pm 150 intensity values ( $\pm 4 \%$ of the total intensity range), the SFT volume changes by approximately $\pm 0.6 \times 10^{6}$ voxels, indicating an error of less than $10 \%$. As a side-effect, the small plateau in the low-intensity threshold region indicates the total body volume.

The homogenisation by diffusion filtering had the advantage that the plateaus were more pronounced and the variability between single slices was reduced (e.g. caused by motion artefacts, cf. Fig. 4b).

In addition, the stability of the segmentation results, derived as a function of the number of iterations employed for the diffusion processing, was investigated. Here, the segmentation for the original datasets was calculated and compared against those generated after 10 and 20 iterations of nonlinear diffusion, respectively. Figure $4 c$ (top panel) shows the differences for the determined fat volume (displayed threshold intensity range, 0-1250) between the original dataset and the dataset after 10 iterations. By increasing the number of iterations, the determination of the fat volume reached stability, indicated by a straight line in Fig. $4 \mathrm{~b}$ (bottom panel).
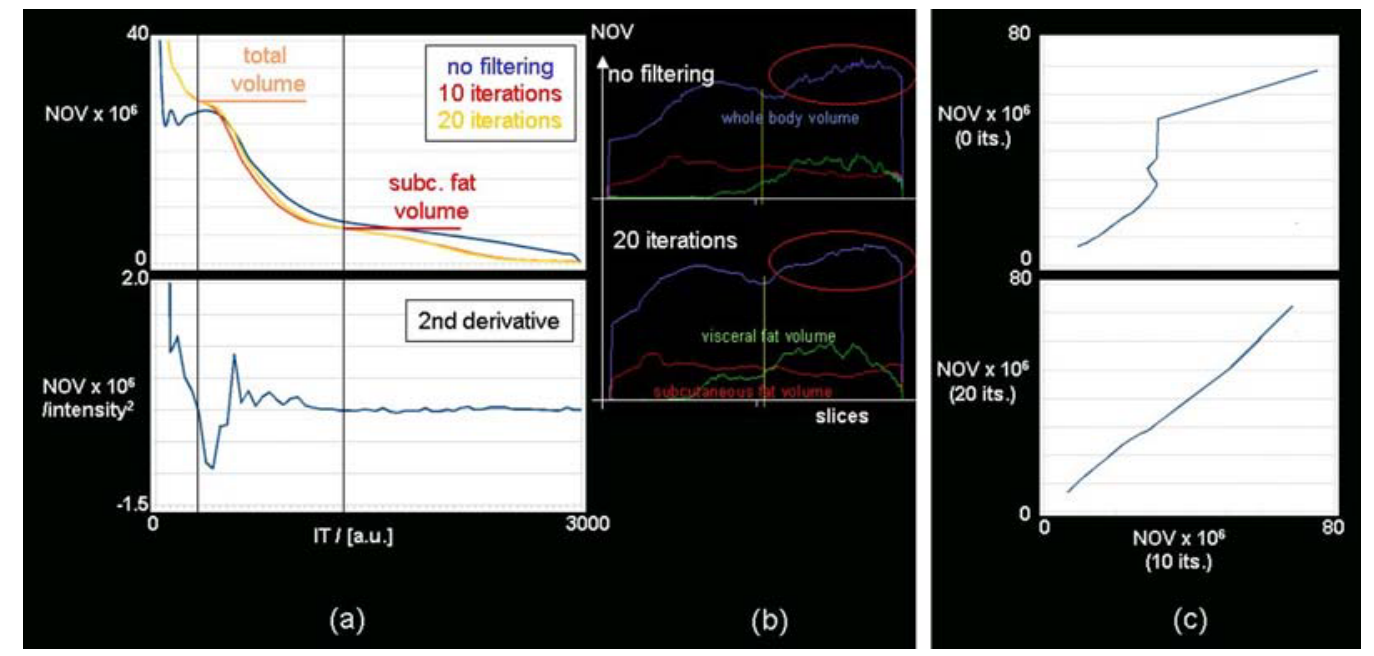

Figure 4. (a) Association between the fat volume and the intensity threshold. The intensity threshold for subcutaneous fat determination was varied in steps of 50 intensity units and the subcutaneous fat tissue (SFT) volume, given as the number of voxels (NOV), was determined (top panel). The characteristics of the ARTIS (Adapted Rendering for Tissue Intensity Segmentation) algorithm revealed stability in the threshold range in which the SFT volume was determined. Here, the second derivative equals zero (bottom panel). As a side-effect, the small plateau in the low-intensity threshold region indicates the total body volume. The histograms show that the segmentation method gives very similar profiles (10 and 20 iterations, respectively). The first plateau (for low-intensity thresholds) indicates the total volume; the second plateau indicates the SFT volume. (b) Homogenisation by diffusion filtering reduces the variability of fat determination among slices caused, for example, by motion artefacts. The bottom panel is extracted from Fig. 4 and juxtaposed to the histogram for nonfiltered data. (c) Stability of the diffusion process in terms of numbers of iterations (its.). Between 0 and 10 iterations, differences could be detected (top panel), Between 10 and 20 iterations, the results appear to be stable (bottom panel). 


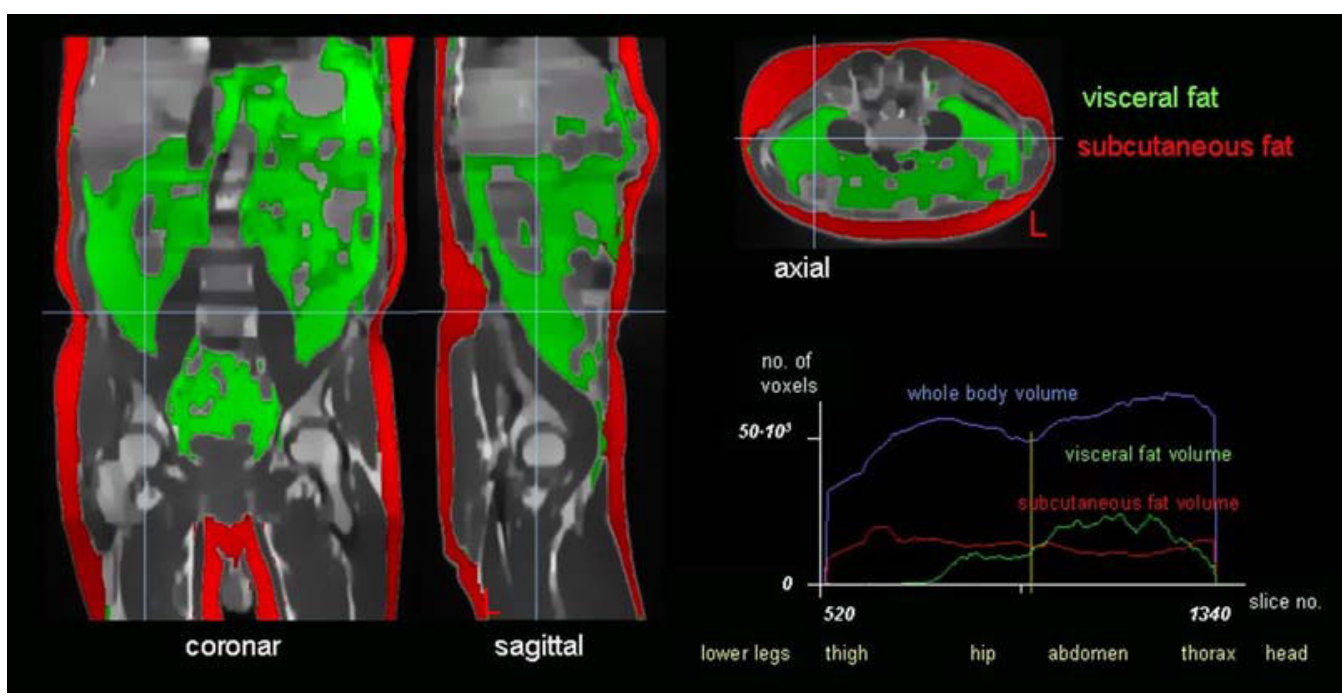

Figure 5. Determination of the fat volume, demonstrated by data from subject 1. In representative slices (coronal, sagittal, axial view), subcutaneous fat tissue (SFT) volume determination is indicated in red and visceral fat tissue (VFT) volume determination is indicated in green. The histogram indicates the fat volume distribution from the thorax to the thighs in the axial direction, i.e. whole-body volume (blue), SFT volume (red) and VFT volume (green). The yellow line indicates an axial slice between hip and abdomen (shown in the top panel).

\section{Segmentation of VFT}

Abdominal VFT was also identified by an operator-defined intensity range selecting all connected voxels with respect to their intensity within the predefined range. Voxels that had been identified previously as SFT were excluded for this search. The remaining voxels were defined via a conventional region grow algorithm, i.e. interconnected voxels with an intensity value in between the thresholds. The result of this operation is displayed in Fig. 5. Alternatively, all regional voxels with an intensity corresponding to subcutaneous fat could be defined as VFT, but the limitation with this technique was that tissues of similar intensities that have no connection to visceral fat were also segmented. As the processing time for the region grow algorithm was on the order of seconds, the use of region grow techniques was preferred.

\section{Analysis of the volume percentage of fat of the whole body and the abdomen}

The whole-body volume was defined via the number of voxels that were above the noise threshold. This threshold could be interactively adjusted for each subject, defining all voxels above the noise level as belonging to the whole-body volume. Thus, the volume percentage of total body fat, SFT volume ratio and VFT volume ratio could be determined.

\section{Additional post-processing}

As additional post-processing opportunities, the standard digital image processing techniques 'opening' and 'closing' are part of ATLAS. The closing procedure defined a voxel as subcutaneous or visceral if at least $90 \%$ of the neighbouring voxels had already been defined as subcutaneous or visceral. The opening procedure worked in the same way, defining a voxel as nonfat if at least $60 \%$ of the neighbouring voxels had already been defined as nonfat. In the pre-processing procedure, the closing technique was applied once after the ARTIS algorithm had been applied.
All algorithms work on the whole three-dimensional volumetric data, as well as on arbitrary two-dimensional slices through this volume, for instance axial slices. An overview through all steps of the applied processing cascade is shown schematically in Fig. 6.

\section{Reproducibility}

Reproducibility was tested on the data from a healthy woman and a healthy man. Three independent MR acquisitions were acquired on 3 days within 1 week. The results for fat volume determination were compared. The threshold dependence is displayed in Fig. 7. Although the results for the male control between the second and third acquisitions were slightly different for the original data, after applying the diffusion algorithm, the SFT volumes were similar (Table 1). The diffusion algorithm showed stable results for 10 relative to 20 iterations. Nevertheless, slight differences could appear (cf. female control on days 2 and 3). Therefore, we decided to apply 20 iteration steps in general, in order to capture all possible variations in the data during processing.

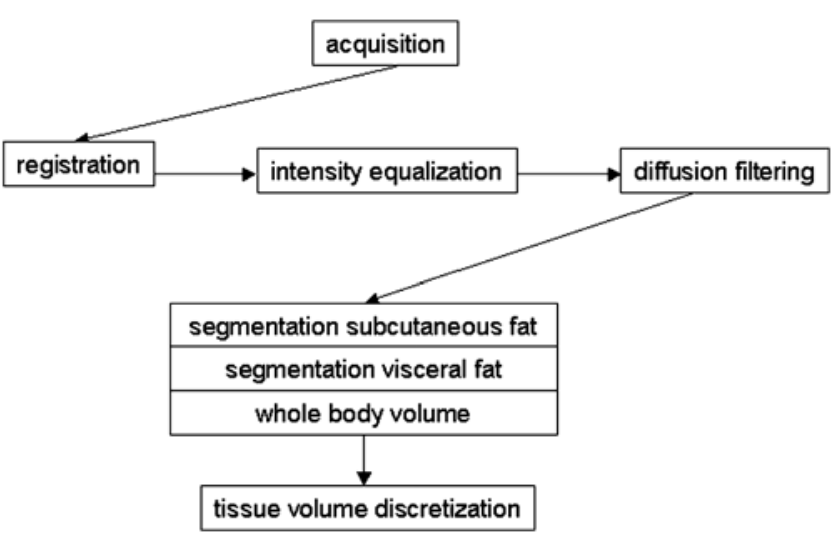

Figure 6. Flowchart of the applied processing cascade. 


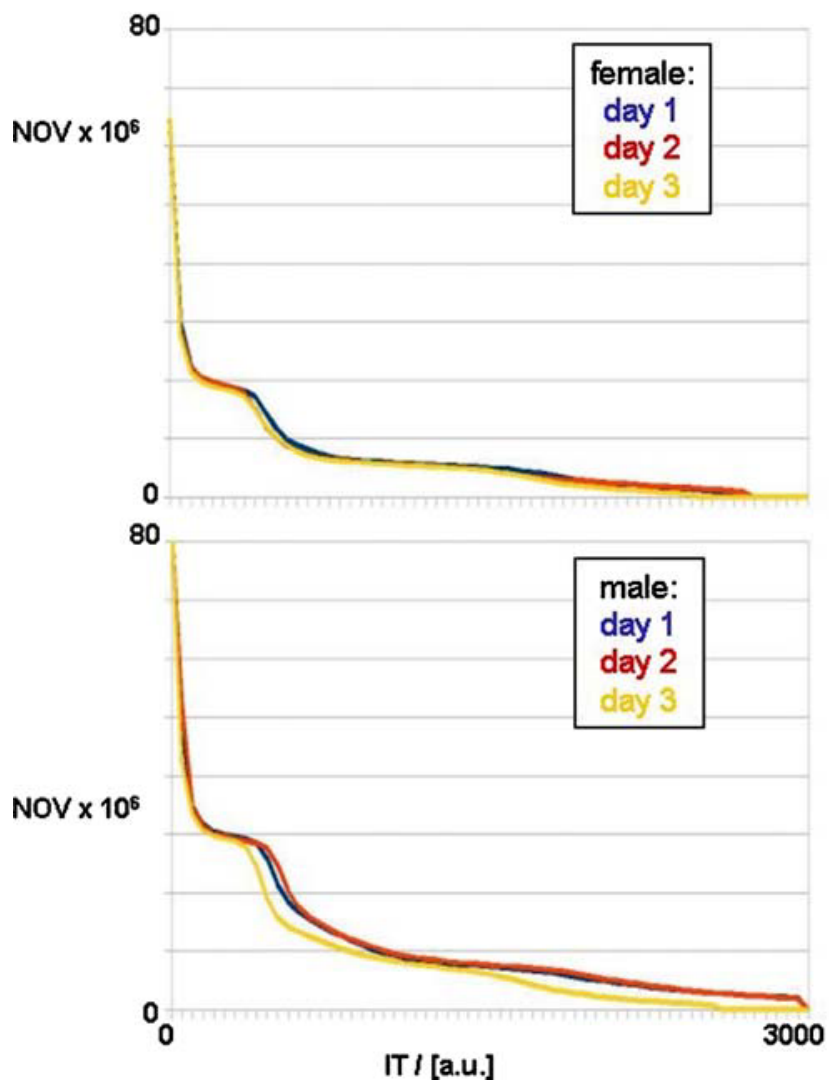

Figure 7. Dependence of the fat volume on the intensity threshold for a male and female control at 3 days within 1 week. Displayed are the dependences for 20 diffusion iterations.

\section{Analysis of healthy controls}

Within the plateaus, the variation in the fat volume was on the order of $\pm 10 \%$. In the centre of the second plateau, in an intensity threshold variation range of $\pm 5 \%$ (three steps of 50 intensity threshold values, i.e. \pm 150 intensity threshold values for the data in this study), the variation of the fat volume was on the order of $\pm 10 \%$. Thus, the total volume and SFT volume could be determined in the centre of the respective plateau (where the second derivative equals zero) with an error of $\pm 10 \%$. The intensity threshold value for the SFT volume could then be used for the VFT volume determination as well. These analyses for the original data, as well as for the data after the application of diffusion filtering (with 10 and 20 iterations), indicated that the histogram analysis remained stable independent of the number of iterations.

The fat volumes for single subjects (Table 1) were calculated from the number of voxels times the voxel size (analysis and display voxel size was $1.2 \times 1.2 \times 1.2 \mathrm{~mm}^{3}$ ). It should be noted that this calculation was based on units of volume, considering the different densities of body tissues and bones; a comparison with units of weight could lead to different percentages.

\section{DISCUSSION}

Interest has grown in the automated detection of fat tissue volumes from whole-body MRI. Recent studies have suggested methods for this task (28-31). Of the techniques proposed to determine body fat tissue percentages from whole-body MRI scans, those suggested in this study provide the ability to reproducibly determine fat tissue with special focus on threshold independence (stability of the results independent of the threshold). The results are in agreement with other investigations measuring the fat volume by MRI $(24-26,36)$ or the percentage of body fat by ultrasonography (37).

In the past, several MRI techniques have been proposed for the measurement of body fat composition and for the quantification of the fat compartments. In most studies, $T_{1}$-weighted imaging (spin- or gradient-echo) has been applied (27-31), in which fat appears hyperintense because of its inherent shorter relaxation time [i.e. $T_{1}$ of fat is approximately $300 \mathrm{~ms}$ in contrast with a value of approximately $1000 \mathrm{~ms}$ for most water-containing tissues (38)]. The composition of subcutaneous and visceral fat is very similar (nearly identical), and it is very difficult to distinguish between the two by chemical shift selective imaging (39) or Dixon techniques (40), which have commonly been proposed to distinguish between fat and water compartments. Functional differences between visceral and subcutaneous adipocytes also seem to be related to their anatomical location (41). Thus, with the methods currently in use, discrimination between SFT and VFT works only by an analysis of their regional distribution. Nevertheless, future efforts in chemical shift imaging might result in the resolution of visceral and subcutaneous fat differences.

The main advantage of MRI-based fat volume determination is that parts or regions of the body can be selected to make the determination more sensitive to fat volume changes caused by medical conditions which affect particular parts of the body.

The ARTIS algorithm was developed to exclusively extract SFT; a different approach has already been suggested by Positano et al. (31). The advantage of ARTIS is that it is possible to detect exclusively subcutaneous fat in regions in which standard region grow techniques fail.

Whole-body MRI scans cannot exclude a lack of intensity caused by local field inhomogeneities or subject movements. Therefore, this approach requires that experts manipulate the lack of intensity via an image repair function. This functionality is implemented as an upscaling of selected regions by a multiplication factor. Therefore, a visual inspection of the results and additional interactive correction by a three-dimensional correction mode are provided. Nevertheless, these corrections are only feasible for small regions.

A different approach has been proposed by Positano et al. (31) to automatically correct for intensity inhomogeneities. Kullberg et al. (28) have reported an automatic and reproducible assessment of adipose tissue from abdominal MRI, as well as the feasibility of automatically assessing whole-body adipose tissue depots from continuously moving bed MRI (29); in addition, a segmentation method for subcutaneous and intermuscular adipose tissue from MRI images of the thigh has been reported (31). The novelty of the present work is that, in addition to these optimised techniques, a universal semiautomated approach, including interactive possibilities, allows for the determination of adipose tissue for all body regions recorded by generally available standard $T_{1}$-weighted imaging. Optimised acquisition techniques, such as the use of Dixontype water and fat imaging, provide a better fat contrast, and the use of thresholding of $T_{1}$-weighted images might, for example, include the whole liver if the fat infiltration is too large. ATLAS is able to manage these fat-optimised datasets, as well as standard $T_{1}$-weighted datasets, by semi-automated applications. 
Table 1. Body fat distribution for the 22 subjects (number of voxels $\times$ voxel size; display voxel size, $1.2 \times 1.2 \times 1.2 \mathrm{~mm}^{3}$ ): body mass index (BMI), total body volume (TV), subcutaneous fat tissue volume (SFTV), visceral fat tissue volume (VFTV) and total fat tissue volume (TFTV)

\begin{tabular}{|c|c|c|c|c|c|c|c|}
\hline Subject/sex & Height $(\mathrm{cm})$ & Weight (kg) & BMI $\left(\mathrm{kg} / \mathrm{m}^{2}\right)$ & TV (L) & SFTV (L) & VFTV (L) & TFTV (L) \\
\hline $1 /$ male & 190 & 97 & 27 & 49.0 & 12.5 & 7.0 & 19.5 \\
\hline 2/male & 183 & 90 & 27 & 49.5 & 13.0 & 5.0 & 18.0 \\
\hline 3/male & 169 & 76 & 27 & 41.0 & 11.0 & 3.0 & 14.0 \\
\hline 4/male & 170 & 85 & 29 & 48.0 & 12.5 & 8.5 & 21.0 \\
\hline $5 /$ male & 175 & 75 & 24 & 41.5 & 9.0 & 7.0 & 16.0 \\
\hline $6 /$ male & 180 & 90 & 28 & 49.0 & 11.5 & 6.0 & 17.5 \\
\hline 7/male & 170 & 115 & 40 & 64.5 & 34.5 & 7.5 & 42.0 \\
\hline $8 /$ male & 182 & 88 & 27 & 48.5 & 12.5 & 9.0 & 21.5 \\
\hline 9/male & 160 & 68 & 26 & 35.0 & 8.5 & 5.5 & 14.0 \\
\hline $10 /$ male & 184 & 85 & 25 & 40.0 & 9.5 & 2.5 & 12.0 \\
\hline $11 /$ female & 166 & 67 & 24 & 36.5 & 16.5 & 3.0 & 19.5 \\
\hline $12 /$ female & 168 & 61 & 22 & 34.5 & 10.5 & 1.5 & 12.0 \\
\hline $13 /$ female & 174 & 65 & 21 & 37.0 & 10.5 & 2.0 & 12.5 \\
\hline 14/female & 160 & 76 & 30 & 48.5 & 15.5 & 6.5 & 22.0 \\
\hline $15 /$ female & 166 & 76 & 28 & 42.0 & 20.5 & 2.5 & 23.0 \\
\hline 16/female & 171 & 82 & 28 & 43.0 & 12.5 & 5.0 & 17.5 \\
\hline 17/female & 170 & 71 & 25 & 44.5 & 21.5 & 4.5 & 26.0 \\
\hline 18/female & 164 & 75 & 28 & 44.0 & 19.5 & 5.5 & 25.0 \\
\hline 19/female & 168 & 60 & 21 & 32.0 & 10.5 & 3.5 & 14.0 \\
\hline 20/female & 155 & 57 & 24 & 32.0 & 10.5 & 2.5 & 13.0 \\
\hline$x x$-day1/female & 179 & 64 & 20 & 32.0 & 10.0 & 0.5 & 10.5 \\
\hline$x x$-day $2 /$ female & & & & 32.0 & 9.5 & 0.5 & 10.0 \\
\hline$x x$-day3/female & & & & 32.5 & 9.5 & 1.0 & 10.5 \\
\hline$x y$-day $1 /$ male & 183 & 91 & 27 & 52.5 & 15.5 & 1.5 & 17.0 \\
\hline$x y$-day $2 /$ male & & & & 52.0 & 15.0 & 2.0 & 17.0 \\
\hline$x y$-day $3 /$ male & & & & 52.0 & 14.5 & 2.0 & 16.5 \\
\hline
\end{tabular}

The methods reported in this study for the determination of body fat compartment volumes by MRI measurement can compete with established imaging techniques, such as bioelectrical impedance analysis and bioimpedance spectroscopy, dual energy X-ray absorptiometry, quantitative computed tomography, dilution techniques, air displacement plethysmography, three-dimensional photonic scanning and positron emission tomography [for a detailed review, see Lee and Gallagher (42)]. The advantages of determination by MRI are that it is totally noninvasive and radiation free, and it provides the opportunity to separate different fat compartments with increasing accuracy. A future study aimed at a comparison between different determination methods should be performed.

\section{CONCLUSION}

The task of this study was to develop a tool to determine the subcutaneous and visceral fat volumes in selected body regions. The analysis must be operator independent (as far as possible) and reproducible. The protocol described in this study, including ATLAS, fulfils these requirements.

MRI data analysis was able to determine SFT and VFT percentages using new analysis strategies. Using the technique described, it was possible to detect changes in SFT and VFT percentages of the total body, as well as of selected body regions.
Discrimination between subcutaneous and visceral fat was feasible for a whole-body-based analysis, as well as for separate sections.

Thus, longitudinal changes over the course of a disease affecting body weight might be detected. Further work will be dedicated to the assessment of changes in tissue volume during the longitudinal course of various diseases and conditions affecting body weight/fat tissue quantity.

\section{REFERENCES}

1. Kissebah AH, Vydelingum N, Murray R, Evans DJ, Hartz AJ, Kalkhoff RK, Adams PW. Relation of body fat distribution to metabolic complications of obesity. J. Clin. Endocrinol. Metab. 1982; 54: 254-260.

2. Krotkiewski M, Björntorp P, Sjöström L, Smith U. Impact of obesity on metabolism in men and women. Importance of regional adipose tissue distribution. J. Clin. Invest. 1983; 72: 1150-1162.

3. Després JP, Tremblay A, Thériault G, Pérusse L, Leblanc C, Bouchard C. Relationships between body fatness, adipose tissue distribution and blood pressure in men and women. J. Clin. Epidemiol. 1988; 41: 889-897.

4. Després JP, Allard C, Tremblay A, Talbot J, Bouchard C. Evidence for a regional component of body fatness in the association with serum lipids in men and women. Metabolism, 1985; 34: 967-973.

5. Abate N, Burns D, Peshock RM, Garg A, Grundy SM. Estimation of adipose tissue mass by magnetic resonance imaging: validation 
against dissection in human cadavers. J. Lipid Res. 1994; 35: 1490-1496.

6. Booth RAD, Goddard BA, Paton A. Measurements of fat thickness in man: a comparison of ultrasound, Harpender caliper and electrical conductivity. Br. J. Nutr. 1966; 20: 719-725.

7. Bullen BA, Quaade F, Olessen E, Lund SA. Ultrasonic reflections used for measuring subcutaneous fat in humans. Hum. Biol. 1965; 37: 375-384.

8. Weingand KW, Hartke GT, Noordsy TW, Ledeboer DA. A minipig model of body adipose tissue distribution. Int. J. Obes. 1989; 13: 347-355.

9. Balta PJ, Ward MW, Tomkins AM. Ultrasound for measurement of subcutaneous fat. Lancet, 1981; 1: 504-505.

10. Borkan GA, Hults DE, Cardarelli J, Burrows BA. Comparison of ultrasound and skinfold measurements in assessment of subcutaneous and total fatness. Am. J. Phys. Anthropol. 1982; 58: 307-313.

11. Chan DC, Watts GF, Barrett PH, Burke V. Waist circumference, waistto-hip ratio and body mass index as predictors of adipose tissue compartments in men. Q.J. Med. 2003; 96: 441-447.

12. Machann J, Thamer C, Schnoedt B, Haap M, Haring HU, Claussen CD, Stumvoll M, Fritsche A, Schick F. Standardized assessment of whole body adipose tissue topography by MRI. J. Magn. Reson. Imaging, 2005; 21: 455-462

13. Baumgartner RN, Heymsfield SB, Roche AF, Bernardino M. Abdominal composition quantified by computed tomography. Am. J. Clin. Nutr. 1988; 48: 936-945.

14. Ferland M, Després JP, Tremblay A, Pinault S, Nadeau A, Moorjani S, Lupien PJ, Thériault G, Bouchard C. Assessment of adipose tissue distribution by computed axial tomography in obese women: association with body density and anthropometric measurements. Br. J. Nutr. 1989; 61: 139-148.

15. Staten MA, Totty WG, Kohrt WM. Measurement of fat distribution by magnetic resonance imaging. Invest. Radiol. 1989; 24: 345-349.

16. Fowler PA, Fuller MF, Glasbey CA, Cameron GG, Foster MA. Validation of the in vivo measurement of adipose tissue by magnetic resonance imaging of lean and obese pigs. Am. J. Clin. Nutr. 1992; 56: 7-13.

17. Gerard EL, Snow RC, Kennedy DN, Frisch RE, Guimaraes AR, Barbieri RL Sorensen AG, Egglin TK, Rosen BR. Overall body fat and regional fat distribution in young women: quantification with MR imaging. Am. J. Roentgenol. 1991; 157: 99-104.

18. Ross R, Shaw KD, Martel Y, de Guise J, Avruch L. Adipose tissue distribution measured by magnetic resonance imaging in obese women. Am. J. Clin. Nutr. 1993; 57: 470-475.

19. Ross R, Shaw KD, Martel Y, de Guise J, Hudson R, Avruch L. Determination of total and regional adipose tissue distribution by magnetic resonance imaging in android women. Basic Life Sci. 1993; 60: 177-180.

20. Cypess AM, Lehman S, Williams G, Tal I, Rodman D, Goldfine AB, Kuo FC, Palmer EL, Tseng YH, Doria A, Kolodny GM, Kahn CR. Identification and importance of brown adipose tissue in adult humans. N. Engl. J. Med. 2009; 360: 1509-1517.

21. van Marken Lichtenbelt WD, Vanhommerig JW, Smulders NM, Drossaerts JM, Kemerink GJ, Bouvy ND, Schrauwen P, Teule GJ. Cold-activated brown adipose tissue in healthy men. N. Engl. J. Med. 2009; 360: 1500-1508.

22. Virtanen KA, Lidell ME, Orava J, Heglind M, Westergren R, Niemi T, Taittonen M, Laine J, Savisto NJ, Enerbäck S, Nuutila P. Functional brown adipose tissue in healthy adults. N. Engl. J. Med. 2009; 360: 1518-1525.

23. Poon CS, Szumowski J, Plewes DB, Ashby P, Henkelman RM. Fat/water quantitation and differential relaxation time measurement using chemical shift imaging technique. Magn. Reson. Imaging, 1989; 7: 369-382.

24. Caprio S, Hyman LD, McCarthy S, Lange R, Bronson M, Tamborlane WV. Fat distribution and cardiovascular risk factors in obese adolescent girls: importance of the intraabdominal fat depot. Am. J. Clin. Nutr. 1996; 64: 12-17.

25. Poll L, Wittsack HJ, Willers R, Mödder U, Heinemann L, Kapitza C, Rave K. Correlation between anthropometric parameters and abdominal fat volumes assessed by a magnetic resonance imaging method in patients with diabetes. Diabetes Technol. Ther. 2004; 6: 844849.

26. Siegel MJ, Hildebolt CF, Bae KT, Hong C, White NH. Total and intraabdominal fat distribution in preadolescents and adolescents: measurement with MR imaging. Radiology, 2007; 242: 846-856.

27. Bonekamp S, Ghosh P, Crawford S, Solga SF, Horska A, Brancati FL, Diehl AM, Smith S, Clark JM. Quantitative comparison and evaluation of software packages for assessment of abdominal adipose tissue distribution by magnetic resonance imaging. Int. J. Obes. 2008; 32: 100-111.

28. Kullberg J, Ahlström H, Johansson L, Frimmel H. Automated and reproducible segmentation of visceral and subcutaneous adipose tissue from abdominal MRI. Int. J. Obes. 2007; 31: 18061817.

29. Kullberg J, Johansson L, Ahlström $H$, Courivaud F, Koken P, Eggers $H$, Börnert P. Automated assessment of whole-body adipose tissue depots from continuously moving bed MRI: a feasibility study. J. Magn. Reson. Imaging, 2009; 30: 185-193.

30. Positano V, Christiansen T, Santarelli MF, Ringgaard S, Landini L, Gastaldelli A. Accurate segmentation of subcutaneous and intermuscular adipose tissue from MR images of the thigh. J. Magn. Reson. Imaging, 2009; 29: 677-684.

31. Positano V, Cusi K, Santarelli MF, Sironi A, Petz R, Defronzo R, Landini L, Gastaldelli A. Automatic correction of intensity inhomogeneities improves unsupervised assessment of abdominal fat by MRI. J. Magn. Reson. Imaging, 2008; 28: 403-410.

32. Press WH, Flannery BP, Teukolsky SA, Vetterling WT. Numerical Recipes in C: The Art of Scientific Computing. Cambridge University Press: New York, 1992; 636-639, 226-228.

33. Alvarez L, Lions P-L, Morel J-M. Image selective smoothing and edge detection by nonlinear diffusion. II. SIAM J. Numer. Anal. 1992; 29: 845-866.

34. Weickert J. Anisotropic Diffusion in Image Processing. Teubner: Stuttgart, 1998.

35. Fick A. Über Diffusion. Ann. Phys. Chem. 1855; 170: 59-86.

36. Poll LW, Wittsack HJ, Koch JA, Willers R, Scherer A, Kapitza C, Heinemann L, Mödder U. Quantification of total abdominal fat volumes using magnetic resonance imaging. Eur. J. Med. Res. 2002; 7: $347-352$.

37. Semiz S, Özgören E, Sabir N, Semiz E. Body fat distribution in childhood obesity: association with metabolic risk factors. Indian Pediatr. 2008; 45: 457-462.

38. Schick F, Eismann B, Jung WI, Bongers $\mathrm{H}$, Bunse M, Lutz O. Comparison of localized proton NMR signals of skeletal muscle and fat tissue in vivo: two lipid compartments in muscle tissue. Magn. Reson. Med. 1993; 29: 158-167.

39. Schick F, Machann J, Brechtel K, Strempfer A, Klumpp B, Stein DT, Jacob S. MRI of muscular fat. Magn. Reson. Med. 2002; 47: 720727.

40. Reeder SB, Pineda AR, Wen Z, Shimakawa A, Yu H, Brittain JH, Gold GE, Beaulieu $\mathrm{CH}$, Pelc NJ. Iterative decomposition of water and fat with echo asymmetry and least-squares estimation (IDEAL): application with fast spin-echo imaging. Magn. Reson. Med. 2005; 54: 636644.

41. Hamdy O, Porramatikul S, Al-Ozairi E. Metabolic obesity: the paradox between visceral and subcutaneous fat. Curr. Diabetes Rev. 2006; 2: 367-373.

42. Lee SY, Gallagher D. Assessment methods in human body composition. Curr. Opin. Clin. Nutr. Metab. Care, 2008; 11: 566-572. 